\title{
Grate Furnace Combustion: A Submodel for the Solid Fuel Layer
}

\author{
H.A.J.A. van Kuijk, R.J.M. Bastiaans, J.A. van Oijen, \\ and L.P.H. de Goey \\ Eindhoven University of Technology, P.O. Box 513, 5600 MB, \\ Eindhoven, The Netherlands \\ h.a.j.a.v.kuijk@tue.nl
}

\begin{abstract}
The reduction of $\mathrm{NO}_{\mathrm{x}}$-formation in biomass fired grate furnaces requires the development of numerical models. To represent the variety in scales and physical processes playing a role in the conversion, newly developed submodels are required. Here, a submodel for the reverse combustion process in the solid fuel layer on the grate is described. The submodel is shown to give good predictions for the velocity of the combustion front as well as for the spatial profiles of porosity, oxygen mass fraction and temperature. These predictions are essential input for $\mathrm{NO}_{\mathrm{x}}$-calculations.
\end{abstract}

Keywords: Grate furnace, reverse combustion, biomass.

\section{Introduction}

A popular way of medium small-scale thermal biomass conversion is the use of grate furnace combustion [1. The operating conditions and design of such a furnace have to be chosen carefully to meet the $\mathrm{NO}_{\mathrm{x}}$ emission limits. Numerical models can support the making of these choices, provided that accurate submodels for the phenomena occurring in the oven are available.

The formation of $\mathrm{NO}_{\mathrm{x}}$ in the furnace (cf. [1]) is strongly determined by the combustion process in the furnace. Generally, the conversion processes takes place in two zones. In the primary conversion zone, the solid fuel is gasified on a moving grate. In the secondary conversion zone, additional air is supplied to burn out the resulting gas mixture. Due to the low combustion temperatures in both zones, the main source for the NO is the fuel-N (fuel-bound nitrogen). In the solid fuel layer, the fuel- $\mathrm{N}$ is released as so-called $\mathrm{N}$-precursors, mainly $\mathrm{NH}_{3}$. In the secondary conversion zone, part of these precursors is converted to NO.

The combustion process as well as the $\mathrm{NO}_{\mathrm{x}}$-formation process is highly complex due to the different physical phenomena involved, i.e. transport of heat and mass as well as chemical reactions. Furthermore, a wide range of length scales is involved. The overall combustion process is determined by the conversion of single particles, occurring at a typical length scale of a particle diameter. Multiple particles are converted simultaneously in a reaction wave, propagating 
trough the solid fuel layer in the primary conversion zone on a length scale of the bed height. Finally, the gases originating from the solid fuel layer burn out and transfer their heat to the boiler present in the oven at a length scale given by the dimension of the oven.

To represent the variety in scales and physical processes affecting the $\mathrm{NO}_{\mathrm{x}}{ }^{-}$ formation process, submodels for (i) the combustion process in the solid fuel layer, (ii) the release of $\mathrm{N}$-precursors from the solid fuel layer (iii) the gas phase combustion process and (iv) the oxidation of the N-precursors are required 2 . Without a submodel for the combustion process in the solid fuel layer, advanced models for the secondary combustion zone (cf. [3]) have to operate with a boundary condition based on measurements just above the solid fuel layer, which strongly restricts their flexibility (cf. [4).

In developing a model for the solid fuel layer, its resemblance to a fixed bed combustion process can be exploited. In a reference frame traveling at the same velocity as the grate, the combustion process is similar to reverse combustion in a fixed bed [5]. In this mode, air is flowing in the upward direction through the fuel layer, while the reaction front propagates downward. Reverse fixed bed combustion processes can be described with 1-dimensional models.

Existing 1-dimensional numerical models for reverse fixed bed combustion (e.g. 6], 7]) and coal have the disadvantage that due to the great amount of detail in the equations and their time-dependent formulation, they require calculation times that are too long to study changes in furnace design and operating conditions [4]. The complexity may also lead to numerical difficulties (cf. [8]). Application of sensitivity analyses to these models is generally not reported, which raises the question up to which extent their complexity and the resulting calculation times are justified.

The present work addresses the need for a model that is able to describe the reaction front propagating in the solid fuel layer with only the essential processes and at low computational costs. Therefore, a limited set of equations based on a model from the literature [9] is used. The predictions of the model are at the length scale of the bed, while in the model chemistry and transport processes at the level of a single particle are accounted for. At the moment the model presented here is operating with a parameter set for coal combustion, but as the combustion process for wood-like biomass is essentially the same, the model can be adapted for this.

In this work, two important new aspects of the model are presented. Firstly, it is shown that this model can be solved also numerically, while in the past it has been solved analytically only [10. This opens the possibility to extend the model for increased accuracy and flexibility if necessary. Secondly, it is shown that stationary solutions for the reverse combustion process can be obtained by using a reference frame attached to the combustion front. This enables convenient performance of parametric studies and results in fast calculation times. In addition, with the numerical analysis it can also be shown that the effect of heat and mass and heat transfer limitations in the fuel bed lead to a change 
in flame structure and thus to the applicability of analytical solution methods. This study is presented elsewhere (cf. [11).

An important feature of the model is that it can be combined with a submodel for the release rates of $\mathrm{N}$-precursors. These release rates are strongly determined by the conversion rate and temperature, (cf. [12]), two quantities that can be predicted with the model. These quantities can serve as input for a submodel consisting of $\mathrm{N}$-precursor release rates based on measurements in a heated grid reactor (cf. [13]) or TGA (cf. [12]))

It is the purpose of this paper to illustrate the advantages of the numerical implementation of the model and the possibilities it offers to serve as a submodel for grate furnace combustion. An extensive comparison of model results with experiments falls not within the present scope. Suffice it to remark that the model currently predicts conversion rates that have the correct order of magnitude compared to experimental results (eg. [14]) which is good, considering that i) the model is not highly detailed, while fixed bed conversion is a complex process, ii) no adaption of the model constants was performed to fit the model to the measurements, iii) there are large uncertainties in some of the model constants and iv) there are large uncertainties in experimental results reported (compare [10] with [14])

The outline of this paper is as follows. First, the model equations are discussed (Sec. 2). Then, it is shown that the model can predict spatial profiles of the variables and the conversion rate of the solid material (Sec. 3). Finally, the conclusions are presented (Sec. 4).

\section{Model Equations and Data}

In this section a short review of the model equations and data presented in Ref. [10 is given. The model consists of a set of three equations for the porosity of the fuel bed $\epsilon$, the temperature $T$ and the oxygen mass fraction $Y$ as a function of the spatial coordinate $x$. In order to obtain this set, the original timedependent model equations have been transformed to a reference frame traveling at the same velocity as the combustion front [9]. This results in:

$$
\begin{gathered}
v_{s} \frac{d}{d x}\left(\rho_{s}(1-\epsilon)\right)=-R \\
v_{s} \frac{d}{d x}\left(\epsilon \rho_{g} Y\right)+\frac{d}{d x}\left(\epsilon \rho_{g} v_{g} Y\right)-\frac{d}{d x}\left(\rho_{g} D \frac{d Y}{d x}\right)=\nu R \\
v_{s} \frac{d}{d x}\left((1-\epsilon) \rho_{s} c_{p s} T+\epsilon \rho_{g} c_{p g} T\right)+\frac{d}{d x}\left(\epsilon \rho_{g} v_{g} c_{p g} T\right)-\frac{d}{d x}\left(\Lambda \frac{d T}{d x}\right)=-\Delta H_{r} R
\end{gathered}
$$

Here, $v_{s}$ is the velocity of the solid, which is moving due to the change in reference frame. The first terms on the rhs. of each equation are convective fluxes involving $v_{s}$. These result from the transformation of time-derivatives in the original reference frame. Other symbols in this set of equations are the reaction 
source term $R$, reaction enthalpy $\Delta H_{r}$, effective coefficients for dispersion $D$ and conduction $\Lambda$ as well as specific heat $c_{p}$ and density $\rho$ for gas and solid (subscripts $g$ and $s$, respectively). The boundary conditions are given at the unburnt side $(u ; x \rightarrow-\infty)$ and burnt side $(b ; x \rightarrow \infty)$

$$
\left.\epsilon\right|_{u}=0,\left.\quad Y\right|_{u}=Y_{u},\left.\quad T\right|_{u}=T_{u} ;\left.\quad \frac{d Y}{d x}\right|_{b}=\left.0 \quad \frac{d T}{d x}\right|_{b}=0 .
$$

In this paper, instead of using $v_{s}$ and $v_{g}$, results are presented in terms of the gas mass flux and solid mass flux at the unburnt side, given by

$$
m_{g u}=\epsilon_{u} \rho_{g u} v_{g u} ; \quad m_{s u}=\left(1-\epsilon_{u}\right) \rho_{s} v_{s u} .
$$

Summarizing, the stationary model equations (112) and (3) together with the boundary conditions can be used to obtain $\epsilon(x), Y(x)$ and $T(x)$ as well as $m_{s u}$ as a a function of gas mass flux $m_{g u}$.

Expressions for the reaction source term, the diffusion coefficient and the conductivity coefficient are required to solve the model. To obtain an expression for $R$ the heterogeneous surface reaction of coal with oxygen

$$
\mathrm{C}+\nu \mathrm{O}_{2} \rightarrow(1+\nu) \mathrm{CO}_{2},
$$

is used. As measurements indicate that the primary product in the reaction zone is $\mathrm{CO}_{2}$ [10, this is a reasonable assumption. The volumetric reaction rate per unit volume can be expressed by

$$
R=\frac{1}{{k_{r}}^{-1}+k_{m}{ }^{-1}} S^{2 / 3} \rho_{g} Y .
$$

Here, $k_{r}$ is an Arrhenius rate constant, $k_{m}$ a mass transfer coefficient and $S$ the specific surface area of the fuel bed. Introducing the dimensionless mass transfer coefficient,

$$
K_{m}=\frac{k_{m}}{k_{r}}
$$

it can be seen that for $K_{m} \rightarrow \infty, R$ is limited by kinetics, while $K_{m} \rightarrow 0$ indicates that $R$ is limited by mass transfer effects. In the kinetically limited case, the expression reduces to that given by Field [15]:

$$
R=k_{r} \rho_{g} Y S .
$$

with $k_{r}$ given by

$$
k_{r}=A_{f} T \exp \left(-\frac{E_{a}}{R T}\right),
$$

in which the pre-exponential factor is indicated with $A_{f}$ and the activation energy by $E_{a}$. The specific surface area in the rate expression can be related to particle properties. For spherical particles of diameter $d_{p}$ in an SC (simple cubic) 
structure reacting as a shrinking core, the specific surface area during conversion can then be expressed by 10 .

$$
S=S_{u}\left(\frac{1-\epsilon}{1-\epsilon_{u}}\right)^{2 / 3} .
$$

Finally, expressions for the gas density, the effective conductivity the dispersion coefficient and the mass transfer coefficient are needed. The gas density is given by

$$
\rho_{g}=\rho_{g u} \frac{T_{u}}{T_{b}} .
$$

Correlations for $\Lambda, D$ and $k_{m}$ are given in [10. The model equations and additional data are implemented in the one dimensional laminar flame code Chem1d [16].

Table 1. Data used for the calculations for a propagating reaction front in a bed of coal particles 10

\begin{tabular}{lll}
\hline Parameter & Unit & Value \\
\hline \hline$T_{u}$ & $\mathrm{~K}$ & 288 \\
$\Delta H$ & $\mathrm{~J} \mathrm{~g}^{-1}$ & $28.9 \times 10^{3}$ \\
$c_{p s}$ & $\mathrm{~J} \mathrm{~kg}^{-1} \mathrm{~K}^{-1}$ & 1.8 \\
$\rho_{s}$ & $\mathrm{~g} \mathrm{~cm}^{-3}$ & $1220 \times 10^{-3}$ \\
$\rho_{s a}$ & $\mathrm{~g} \mathrm{~cm}^{-3}$ & $470 \times 10^{-3}$ \\
$d_{p}$ & $\mathrm{~cm}$ & 2.5 \\
$\rho_{g u}\left(T_{u}\right)$ & $\mathrm{g} \mathrm{cm}^{-3}$ & $1.226 \times 10^{-3}$ \\
$c_{p g}$ & $\mathrm{~J} \mathrm{~kg}^{-1} \mathrm{~K}^{-1}$ & 1.13 \\
$Y_{u}$ & - & 0.23 \\
$A_{f}$ & $\mathrm{~cm} \mathrm{~K}^{-1} \mathrm{~s}^{-1}$ & $6 \times 10^{4}$ \\
$E_{a}$ & $\mathrm{~kJ} \mathrm{~mol}^{-1}$ & 150 \\
$\nu$ & - & 2.67 \\
\hline
\end{tabular}

\section{Results}

Model results consist of spatial profiles of $\epsilon, T$ and the heat release $\dot{q}$. Here, we present solutions for varying $m_{g u}$ (cf. Fig. 1). The latter quantity can be determined by the equation $\dot{q}=-\Delta H_{r} R$ and is a measure for the magnitude reaction source term. The results show that with increasing $m_{g u}, \dot{q}$ increases. At the same time, the width of the boundary layers in the profiles for $T$ and $Y$ increases. Due to the increased flow of air, the conversion of the solid fuel $\epsilon_{b}$ as well as $T_{b}$ become higher.

From the spatial flame profiles for different $m_{g u}$, the conversion $\epsilon_{b}$ as well as $T_{b}$ can be determined as a function of $m_{g u}$ (cf. Figs. 2b, 2r). By using $T_{b}$ in the evaluation of $K_{m}$ (cf. (8) ), $K_{m b}$ can be determined (cf. Fig. 22d). Furthermore, $m_{s u}$ results from the calculations (cf. Fig. 2a). It can be observed that $m_{s u}$ initially increases when $m_{g u}$ increases, until a maximum is reached. Then, $m_{s u}$ 


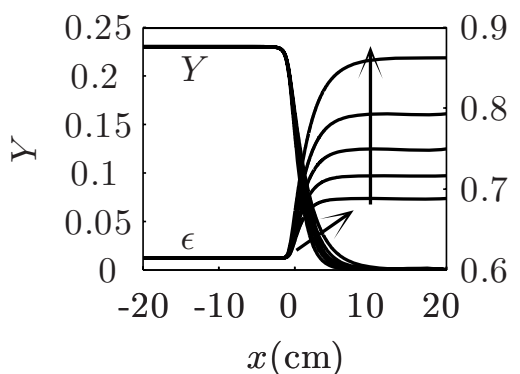

(a)

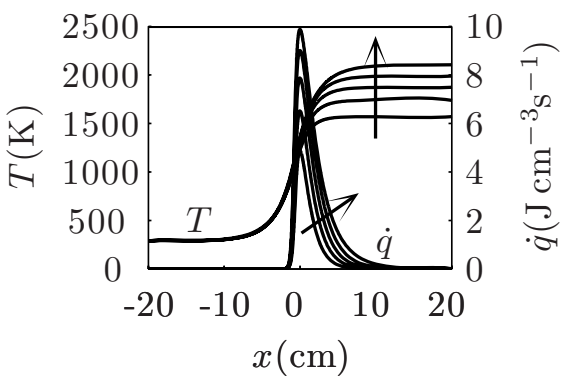

(b)

Fig. 1. Spatial flame profiles for varying gas mass flux. Results for $Y$ and $\epsilon$ (a) and $T$ and $\dot{q}$ (b). The arrows indicate results obtained with increasing $m_{g u}$ from $m_{g u}=$ $4.9 \times 10^{-3} \mathrm{~g} \mathrm{~cm}^{-2} \mathrm{~s}^{-1}$ to $m_{g u}=14.6 \times 10^{-3} \mathrm{~g} \mathrm{~cm}^{-2} \mathrm{~s}^{-1}$ in steps of $2.4 \times 10^{-3} \mathrm{~g} \mathrm{~cm}^{-2} \mathrm{~s}^{-1}$.

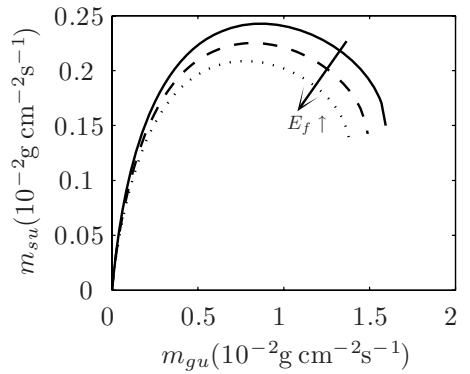

(a)

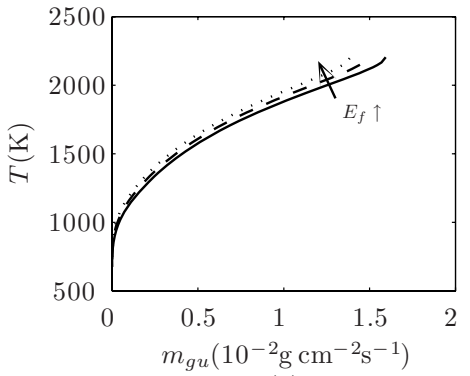

(c)

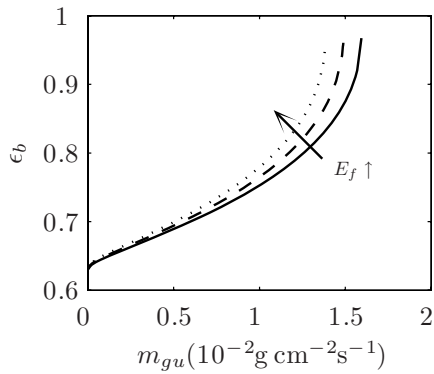

(b)

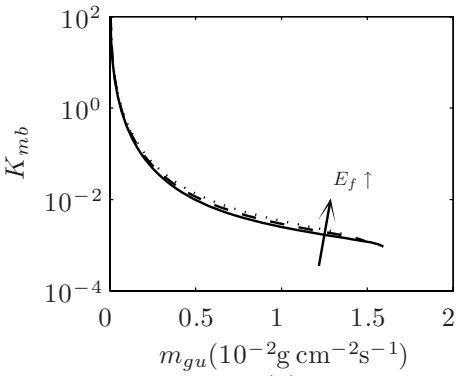

(d)

Fig. 2. Flame parameters as a function of gas mass flux for varying activation energy. Results for the solid mass flux (a), the porosity (b), the temperature (c) and dimensionless mass transfer coefficient (d). Solid line: $E_{a}=150 \mathrm{~kJ} \mathrm{~mol}^{-1}$, dashed line: $E_{a}=160 \mathrm{~kJ} \mathrm{~mol}^{-1}$, dotted line: $E_{a}=170 \mathrm{~kJ} \mathrm{~mol}^{-1}$.

decreases until extinction takes place. The shape of the curve of $m_{s u}$ as a function of $m_{g u}$ (Fig. 2) was explained in earlier works in terms of the counterbalancing effects of the increased oxygen supply to and and increased convective heat transport out of the reaction zone with increasing $m_{g u}$ (cf. [17]). The temperature $T_{b}$ and porosity $\epsilon_{b}$ increase continuously with increasing $m_{g u}$. (Due to limitations 
of the current numerical implementation, the extinction point $\epsilon_{b}=1$ is not reached completely). The dimensionless mass transfer coefficient $K_{m b}$ (i.e. $K_{m}$ evaluated at $T_{b}$ ) shows that for low $m_{g u}$, the source term is controlled by kinetics $\left(K_{m b}>1\right)$. At $m_{g u} \sim 1.0 \times 10^{-3} \mathrm{~g} \mathrm{~cm}^{-2} \mathrm{~s}^{-1}$ mass transfer effects begin to play a role $\left(K_{m b}<1\right)$. Furthermore, it can be seen that all parameters are sensitive to changes in $E_{a}$.

A typical calculation time to obtain results for a variation of $m_{g u}$ from zero up to the extinction point is only ten minutes on a PC. Furthermore, with other models for coal beds with an instationary formulation (e.g. [18]), it is no longer necessary to wait until a steady state is reached when a parameter is varied.

\section{Conclusions}

From the numerical results presented in this paper, it can be concluded that the two most important quantities that determine the release of the N-precursors can be predicted, namely the front velocity and temperature. These quantities can be determined as a function of gas mass flow, the main controlling parameter in a grate furnace. In the calculations, both kinetics and mass transfer limitations can be taken in into account. Furthermore, the effect of fuel parameters (particle size and bed density) that show strong variations in practical combustion processes has been included via the correlations used to determine the model coefficients.

The implementation of a stationary set of equations as is performed here has two important advantages. Firstly, the calculation times are short. This makes the method proposed here suitable for application as a submodel in a grate furnace. Moreover, the the model can be extend further with additional important phenomena and still remain within the limits of reasonable calculation times. In addition, the instationary implementation makes it convenient to perform parametric studies. This makes it achievable to study the effect of additional phenomena included in the model by means of a sensitivity analysis to determine their effect on the velocity of the reaction front, the temperature and the structure of the reaction zone.

First, the model will be extended to describe the additional chemistry necessary for biomass conversion by using the Chem1D modules for the gas phase kinetics. It is also planned to include the effect of other parameters that are shown to have a large impact on the combustion process in practical applications, e.g. the moisture content of the fuel.

\section{References}

1. S. van der Loo, H. Koppejan, Handbook of Biomass: Combustion and Cofiring, Twente University Press, 2002.

2. R. J. M. Bastiaans, H. A. J. A. van Kuijk, B. A. Albrecht, J. A. van Oijen, L. P. H. de Goey, Catalysis for Renewables, Wiley-VCH, 2006, Ch. Thermal biomass conversion and NOx emissions. 
3. B. A. Albrecht, R. J. M. Bastiaans, J. A. van Oijen, L. P. H. de Goey, NOx emissions modelling in biomass combustion grate furnaces, in: A. Reis, W. J., W. Leuckel (Eds.), Proceedings of the 7th European Conference on Industial Furnaces and Boilers, Porto, Portugal, 2006.

4. R. Scharler, T. Feckl, I. Obernberger, Modification of a Magnussen constant of the Eddy Dissipation Model for biomass grate furnaces by means of hot gas in-situ FT-IR absorption spectroscopy, Progress in Computational Fluid Dynamics 3 (2-4) (2003) 102-111.

5. H. Thunman, B. Leckner, Ignition and propagation of a reaction front in crosscurrent bed combustion of wet biofuels, Fuel 80 (4) (2001) 473-481.

6. H. Thunman, B. Leckner, Influence of size and density of fuel on combustion in a packed bed, Proceedings of the Combustion Institute 30 (2) (2005) 2939-2946.

7. J. C. Wurzenberger, S. Wallner, H. Raupenstrauch, J. G. Khinast, Thermal conversion of biomass: Comprehensive reactor and particle modeling, AIChE Journal 48 (10) (2002) 2398-2411.

8. P. T. Radulovic, M. U. Ghani, L. D. Smoot, An improved model for fixed bed coal combustion and gasification, Fuel 74 (4) (1995) 582-594.

9. R. Gort, J. J. H. Brouwers, Theoretical analysis of the propagation of a reaction front in a packed bed, Combust. Flame 124 (1-2) (2001) 1-13.

10. R. Gort, On the propagation of a reaction front in a packed bed, Ph.D. thesis, Twente University, Enschede, The Netherlands (1995).

11. H. A. J. A. van Kuijk, R. J. M. Bastiaans, J. A. Oijen, L. P. H. de Goey, Reverse fixed bed combustion: kinetically controlled and mass transfer controlled flame structures. To be published.

12. W. de Jong, A. Pirone, M. A. Wojtowicz, Pyrolysis of miscanthus giganteus and wood pellets: TG-FTIR analysis and reaction kinetics, Fuel 82 (9) (2003) 1139 1147.

13. A. Toland, R. J. M. Bastiaans, A. Holten, L. P. H. Goey, Kinetics of CO release from bark and medium density fibre board pyrolysis, Biomass and Bioenergy. Submitted for publication.

14. P. Nicholls, Underfeed combustion, effect of preheat, and distribution of ash in fuel beds, Bulletin of the United States Bureau of Mines 378 (1934) 1-76.

15. M. A. Field, D. W. Gill, B. B. Morgan, P. G. W. Hawksly, Combustion of Pulverized Coal, BCURA, Leatherhead, 1967.

16. Chem1d, A one-dimensional laminar flame code. www.combustion.tue.nl.

17. D. Lozinski, J. Buckmaster, Quenching of reverse smolder, Combust. Flame 102 (12) (1995) 87-100.

18. J. Cooper, W. L. H. Hallett, A numerical model for packed-bed combustion of char particles, Chemical Engineering Science 55 (20) (2000) 4451-4460. 